\title{
Bimeristina binnekampi n. g., n. sp., MERISTELOIDEO (BRAQUIÓPODO) DEL DEVÓNICO INFERIOR (PRAGUIENSE) DE ESPAÑA
}

\author{
Jenaro L. GARCÍA-ALCALDE \\ Departamento de Geología (Paleontología), Universidad de Oviedo. E- \\ mail: jalcalde@geol.uniovi.es.
}

\begin{abstract}
García-Alcalde, J.L. 2003. Bimeristina binnekampi n. g., n. sp., meristeloideo (braquiópodo) del Devónico Inferior (Praguiense) de España. [Bimeristina binnekampi n. g., n. sp., a Lower Devonian (Pragian) meristelloid (brachiopod) from Spain.] Revista Española de Paleontología, 18(1), 103-112. ISSN 0213-6937.
\end{abstract}

\begin{abstract}
A new meristelloid genus, Bimeristina, based in the new species B. binnekampi is described and figured. This species occurs in Pragian distal platform, carbonatic rocks of the so called Cantabro-Celtiberian region (Cantabrian Mountains, Palentian Domain, upper E member of the Lebanza Formation; eastern Guadarrama, Ce3 to $\mathrm{Ce} 4$ members of the Cercadillo Formation; and eastern Iberian Cordillera, Aragon, d2c $\alpha$ to d $2 \mathrm{c} \beta$ members of the Nogueras Formation, Spain). The new genus and species are characterized by a Meristella jugal apparatus supplemented with the jugal arch connected both with the primary and the secondary lamellae of the spiral cones, and septalium with a more or less developed median ridge.
\end{abstract}

Keywords: Brachiopoda, meristelloids, jugal apparatus, Pragian, Cantabro-Celtiberian zone, Spain.

\section{RESUMEN}

Se describe y figura un nuevo género de braquiópodo Rhynchonellata meristeloideo, Bimeristina, basado en la nueva especie $B$. binnekampi. El nuevo taxon se distribuye a lo largo del borde distal de una plataforma carbonatada desarrollada durante el Devónico Inferior (Praguiense) en la llamada región Cántabro-Celtibérica (Cordillera Cantábrica, Dominio Palentino: parte superior del miembro E de la Formación Lebanza; Guadarrama oriental: miembros $\mathrm{Ce} 3$ y Ce4 de la Formación Cercadillo; Cordillera Ibérica oriental, Aragón: transición entre los miembros $\mathrm{d} 2 \mathrm{c} \alpha-\mathrm{d} 2 \mathrm{c} \beta$, de la Formación Nogueras). Esta nueva forma se caracteriza principalmente por combinar estructuras yugales similares a las de Meristella con un arco yugal en doble conexión con las lamelas primarias y secundarias del espiralio, inexistentes en dicho género y en el resto de los de la Subfamilia Meristellinae, y también por poseer un septalio en cuyo fondo se encuentra una elevación media, más o menos desarrollada.

Palabras clave: Braquiópodos, meristeloideos, aparato yugal, Praguiense, región Cántabro-Celtibérica, España.

\section{INTRODUCCIÓN}

En España las faunas del Praguiense son comparativamente menos conocidas que las de otros períodos del Devónico Inferior. En lo que concierne a la Zona Cantábrica, en particular, esta peculiaridad se explica por las condiciones geológicas de la época, con predominio de niveles marinos someros en la transición Lochkoviense-Praguiense, que originaron depósitos dolomíticos frecuentes, que representan condiciones adversas para el registro de faunas conchíferas abundantes (García-Alcalde, 1999).

Sin embargo, la franja más distal de la plataforma de la época mantuvo profundidades adecuadas para el desarrollo de faunas diversas. Esto se aprecia con claridad en el Dominio Palentino ( $N$ de España), en las Calizas de Lebanza, del Lochkoviense y Praguiense (Fig. 1). Varias publicaciones anteriores (Binnekamp, 1965; Álvarez, 1990; García-Alcalde, 1998, 1999; GarcíaAlcalde et al., 1990, 2002; García-López et al., 2002) dieron cuenta de la notable variedad y abundancia de faunas de braquiópodos en la referida formación, abarcando los intervalos faunísticos 2 a 6 de GarcíaAlcalde (1996). En particular, las faunas de braquiópodos tuvieron un notable desarrollo en la parte más alta de la formación, en el llamado miembro E (Fig. 1), compuesto por calizas packstone y wackestone con intercalaciones de pizarras oscuras hacia el techo, depositadas en un 


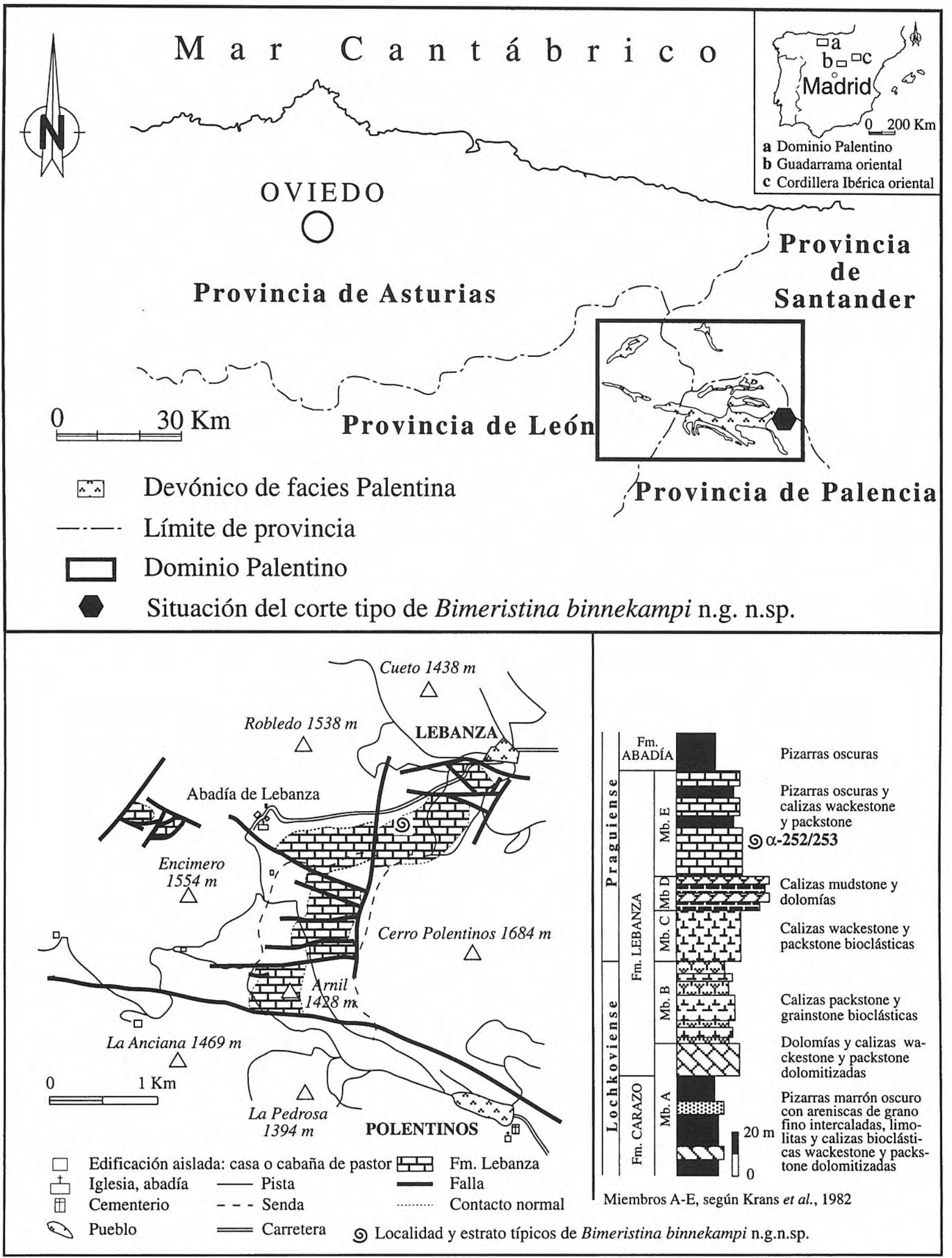

Figura 1. Situación geográfica y estratigráfica del área, localidad y estrato típicos de Bimeristina binnekampi n. g., n. sp. Geographical and stratigraphical situation of the type area, locus and stratum typicum of Bimeristina binnekampi $n$. g., n. sp. 


\begin{tabular}{lcccccccc}
\multicolumn{1}{c}{ Ejemplar } & $\mathrm{L}$ & $\mathrm{a}$ & $\mathrm{g}$ & $\mathrm{a} / \mathrm{L}$ & $\mathrm{g} / \mathrm{L}$ & $\mathrm{Aa}$ & $\mathrm{Dsd}$ & Dam \\
Holotipo DPO30985 & 23,4 & 23 & 15 & 0,98 & 0,65 & $88^{\circ}$ & 62 & 53 \\
Paratipo DPO 30868 & 12,3 & 11,9 & 6,7 & 0,97 & 0,54 & $97^{\circ}$ & 60 & 55 \\
Paratipo DPO 30876 & 21,1 & 21,6 & 11,9 & 1,02 & 0,56 & $96^{\circ}$ & 69 & 55 \\
Paratipo DPO 30887 & 16,8 & 15,6 & 9,6 & 0,93 & 0,57 & $95^{\circ}$ & 52 & 57 \\
Paratipo DPO 30888 & 18,3 & 18,3 & 10,3 & 1 & 0,56 & $102^{\circ}$ & 53 & 54 \\
Paratipo DPO 30889 & 17,2 & 17 & 10,2 & 0,99 & 0,60 & $94^{\circ}$ & 57 & 56 \\
Paratipo DPO 30890 & 16,1 & 14,1 & 7,8 & 0,87 & 0,48 & $92^{\circ}$ & 61 & 53 \\
Paratipo DPO 30891 & 18,6 & 17,1 & 12,6 & 0,92 & 0,68 & $83^{\circ}$ & 53 & 59 \\
Paratipo DPO 30892 & 12,1 & 10,6 & 6,9 & 0,88 & 0,56 & $93^{\circ}$ & 53 & 56 \\
Paratipo DPO 30893 & 14,8 & 15 & 8,7 & 1,01 & 0,59 & $119^{\circ}$ & 65 & 51 \\
Paratipo DPO 30894 & 11 & 10,2 & 6,2 & 0,93 & 0,56 & $87^{\circ}$ & 58 & 60 \\
Paratipo DPO 30980 & 13,6 & 13,2 & 7,2 & 0,97 & 0,58 & $93^{\circ}$ & 62 & 53 \\
DPO 30856 & 22,3 & 20,3 & 15,7 & 0,91 & 0,70 & $80^{\circ}$ & 62 & 56 \\
DPO 30860 & 20,7 & 21,6 & 13,9 & 1,04 & 0,67 & $96^{\circ}$ & 66 & 55 \\
DPO 31348 & 14,6 & 13,8 & 7,7 & 0,94 & 0,53 & $90^{\circ}$ & 58 & 58 \\
DPO 38571 & 17,9 & 16,6 & 10,2 & 0,93 & 0,57 & $91^{\circ}$ & 59 & 54 \\
DPO 38572 (Seccionado) & 17 & 16,6 & 9,5 & 0,97 & 0,58 & $100^{\circ}$ & 55 & 58
\end{tabular}

L, a, g = Longitud, anchura y grosor de la concha, respectivamente; Aa = Angulo apical; Dam = Situación de la máxima anchura de la concha expresada como porcentaje de L; Dsd = Longitud del septo medio dorsal expresada como porcentaje de la longitud de la valva.

$L, a, g=$ Shell length, width and thickness, respectively; Aa=Apical angle; Dam=Situation of the shell width expressed as percent of shell length; Dsd=length of the dorsal median septum expressed as percent of dorsal valve length.

Tabla 1. Principales medidas de los 18 ejemplares mejor conservados de B. binnekampi n. g., n. sp. (L, a, g, expresados en mm. Ver también Fig. 6).

Most relevant parameters of the 18 best preserved specimens of B. binnekampi $n . g ., n$. sp. (L, a, g, are in mm. See also Fig. 6).

ambiente marino, abierto y tranquilo en el curso de un período de hundimiento epirogénico generalizado (Krans et al., 1982). El miembro E corresponde a la Biozona de conodontos Icriodus simulator, del Praguiense Inferior, según García-López et al. (2002).

En el miembro E de la Fm. Lebanza se encontraron muchas formas nuevas del Phylum Brachiopoda. En este trabajo, se analiza un curioso representante de la Familia Meristellidae, caracterizado por la posesión de un aparato braquial muy original. Esta forma constituye una de las más características del Intervalo Faunístico 6 (GarcíaAlcalde, 1996) en Palencia y hasta ahora no pudo identificarse en otras áreas de la Zona Cantábrica, como Asturias o León. En cambio, representantes del mismo género y especie aparecen en la Cordillera Ibérica oriental (Aragón), en capas de la parte superior de la Fm. Nogueras, y en el Guadarrama oriental, también en capas superiores de la Fm. Cercadillo (ver versión actualizada de la estratigrafía de las regiones referidas en GarcíaAlcalde et al., 2002). Esto contribuye a resaltar más la unidad esencial de la región Cántabro-Celtibérica (Cordillera Cantábrica, Guadarrama oriental y Aragón) (García-Alcalde, 1999).

\section{SISTEMÁTICA}

El material que se describe a continuación, registrado con las siglas DPO, está depositado en las colecciones del Departamento de Geología de la Universidad de Oviedo. El siglado RGM está depositado en el Rijksmuseum van Geologie en Mineralogie de Leiden (Holanda).

PHYLUM BRACHIOPODA Duméril, 1806 SUBPHYLUM RHYNCHONELLIFORMEA Williams et al., 1996

CLASE RHYNCHONELLATA Williams et al., 1996

ORDEN ATHYRIDIDA Boucot, Johnson y Staton, 1964, emend. Álvarez, Rong y Boucot, 1998.

SUBORDEN ATHYRIDIDINA Boucot, Johnson y

Staton, 1964, emend. Álvarez, Rong y Boucot, 1998

Superfamilia Meristelloidea Waagen, 1883

Familia Meristellidae Waagen, 1883

Subfamilia Meristellinae Waagen, 1883

\section{Observaciones}

Los meristélidos, en particular las formas próximas a Meristella Hall, 1859, fueron caracterizados por la 


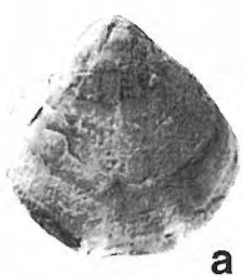

a
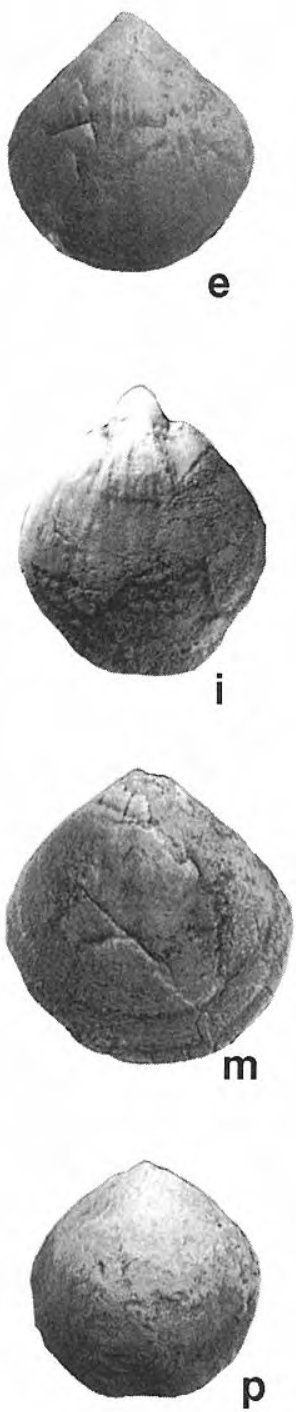
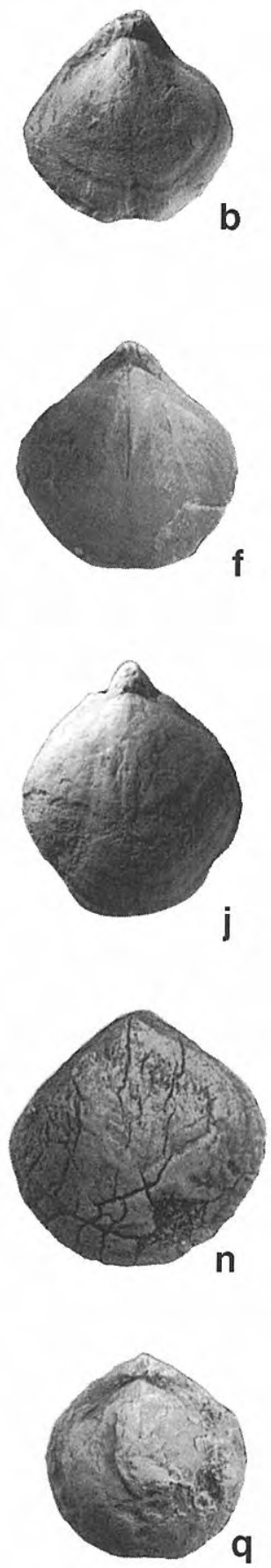
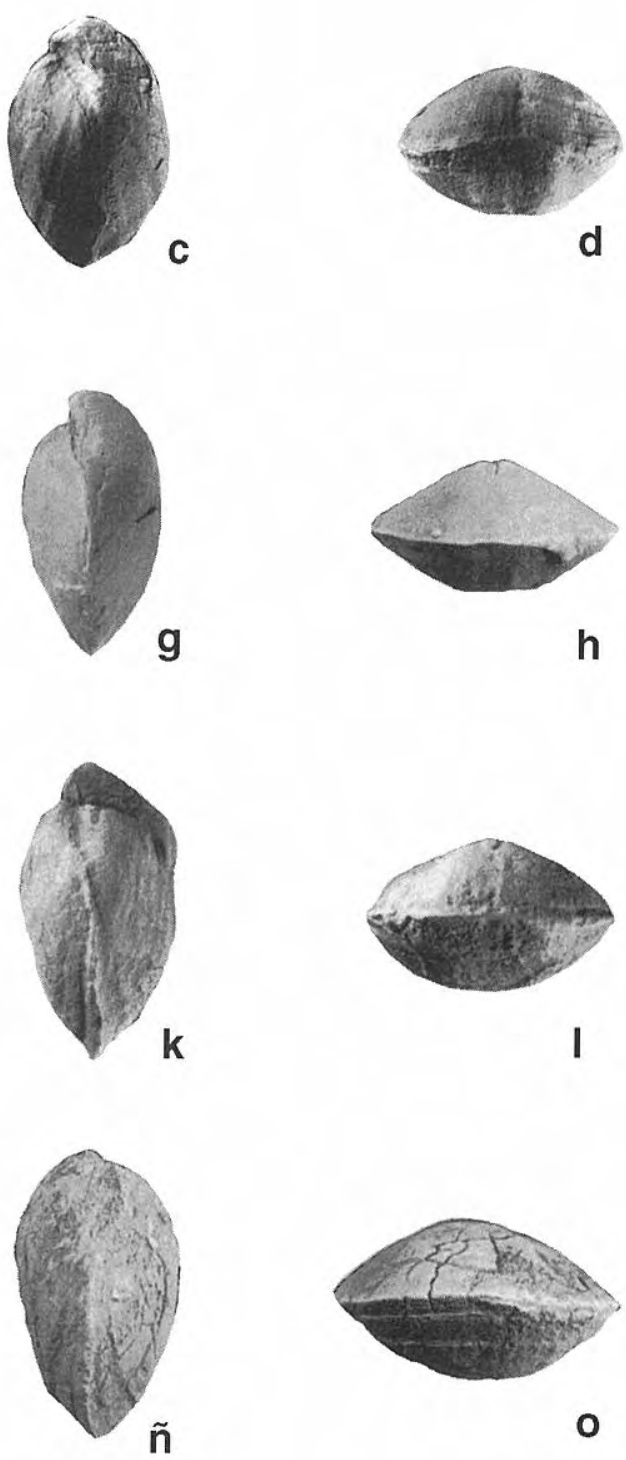

0
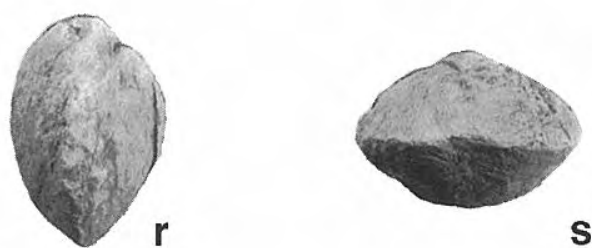

Figura 2. Bimeristina binnekampi n. g., n. sp. a-d: Holotipo DPO 30895, vistas ventral, dorsal, lateral y anterior, x1. e-h: Paratipo DPO 30980, vistas ventral, dorsal, lateral y anterior de un ejemplar algo desconchado mostrando en $\mathrm{f}$ la traza del largo septo medio dorsal, x2. i-l: Paratipo DPO 30863, vistas ventral, dorsal, lateral y anterior de un ejemplar completamente desconchado mostrando ambos campos musculares, x2. m-o: Ejemplar DPO 38577, vistas ventral, dorsal, lateral y anterior, x2. Guadarrama oriental, miembros C3 a C4 (=nivel MS 11/12=A-149=A-201) de la Fm. Cercadillo. p-s: Ejemplar geróntico DPO 33969, vistas ventral, dorsal, lateral y anterior, x1. Cordillera Ibérica Oriental, transición entre los miembros d2c $\alpha$ y d2c $\beta$ (nivel A-133) de la Fm. Nogueras.

a-d: Holotype DPO 30985, ventral, dorsal, lateral and anterior views, xl. e-h: Paratype DPO 30980, ventral, dorsal, lateral and anterior views of a decorticated specimen showing in $f$ the long dorsal median septum, $x 2 . i-l$ : Paratype DPO 30863, ventral, dorsal, lateral and anterior views of an entirely decorticated specimen showing the muscle fields of both valves, x1. m-o: Specimen DPO 38577, ventral, dorsal, lateral and anterior views, x2. Eastern Guadarrama, C3-C4 members (bed MS 11/12=A-149=A-201) of the Cercadillo Fm. p-s: Aged specimen DPO 33969, ventral, dorsal, lateral, and anterior views, x1. Eastern Iberian Cordillera, $d 2 c \alpha-d 2 c \beta$ members transition (bed A-133), of the Nogueras Fm. 

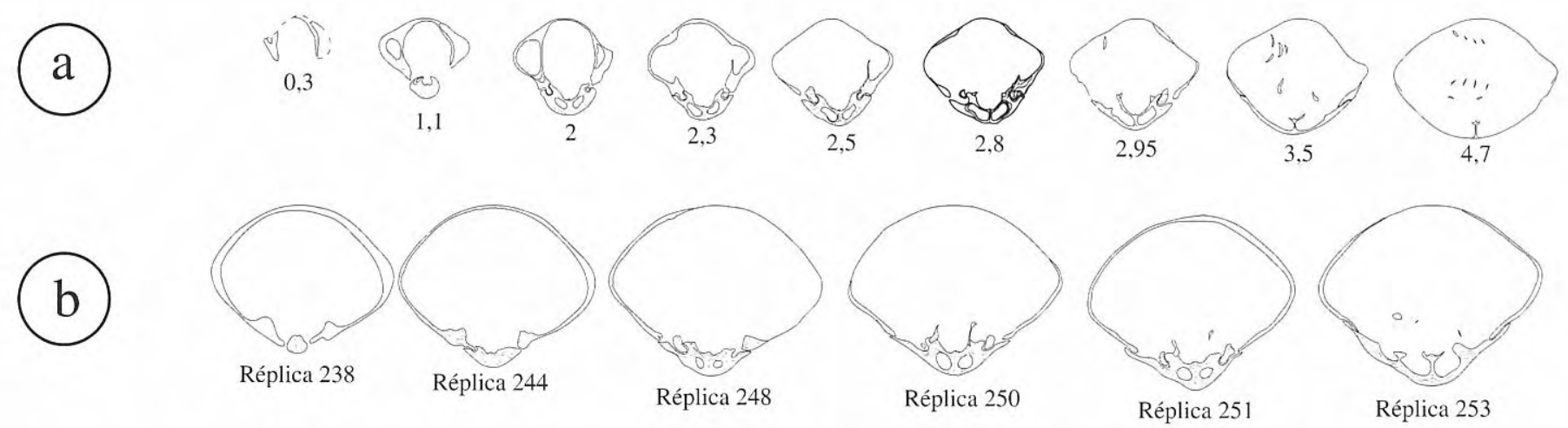

Figura 3. Bimeristina binnekampi n. g., n. sp. Devónico Inferior (Praguiense). Dibujos con cámara clara (aprox. x2) de secciones perpendiculares a los planos comisural y de simetría. a: Ejemplar DPO 38570, yacimiento $\alpha-176$ de la Fm. Lebanza (miembro E), cerca del pueblo de Lebanza (Dimensiones: L=17,5 mm, a=17,2 mm, g=10,1 mm). Distancias en $\mathrm{mm}$ al ápice de la valva ventral.- El braquidio y yugo del ejemplar estaban dañados. b: Ejemplar RGM 360.055, de la colección original de Binnekamp (1965), depositado en el Museo de Leiden, etiquetado como "Whitfieldella" spec. No constan procedencia ni dimensiones del ejemplar. Serie muy incompleta que no llegaba hasta el braquidio y yugo.

Lower Devonian (Pragian). Camera lucida drawings (ca. x2) of serial sections normal to the commisural and symmetry planes. Brachidium and jugum broken. a: Specimen DPO 38570, locality $\alpha-176$ of the Lebanza Fm (Mb $E$ ), near the village of Lebanza (Palencia, $N$ Spain). (Dimensions: $L=17.5 \mathrm{~mm}, a=17.2 \mathrm{~mm}, \mathrm{~g}=10.1 \mathrm{~mm}$ ). Distances measured in $\mathrm{mm}$ to the ventral valve apex. $\boldsymbol{b}$ : Specimen RGM 360055, of Binnekamp's (1965) original collection, labeled "Whitfieldella" spec. and housed in the Rijkmuseum of Leiden. Detailed occurrence and dimensions of the specimen unknown. Very incomplet series that is not reaching the brachidium and jugum.

carencia de expansiones conchíferas lamelares a lo largo de las líneas de crecimiento, presencia de placas dentales subparalelas, septalio no cubierto, apoyado en un septo medio, más o menos largo, y un aparato braquial complejo, con estructuras yugales a veces muy elaboradas (Álvarez et al., 1998). El grupo se originó a finales del Silúrico y tuvo su momento de máxima expansión a lo largo del Devónico, cuando aparecieron numerosos géneros que colonizaron, en general, medios marinos de baja energía, cerca de los bordes de las plataformas en casi todo el mundo.

Álvarez et al. (1998) estiman que la familia no llegó a desarrollar silla yugal en el yugo y, por otro lado, agrupan en la Subfamilia Meristellinae formas con arco yugal proyectado posteriormente en un largo tallo que puede bifurcarse en ramas yugales accesorias, a veces formando volutas que vuelven a unirse al tallo. El nuevo género, que se describe a continuación, posee un arco yugal que se prolonga anteriormente en dos cortas proyecciones simulando una silla yugal incipiente y el yugo comprende, además de un largo tallo yugal y volutas accesorias típicamente meristelinas, unidas anteriormente al tallo, sendas ramas yugales laterales suplementarias, conectando el yugo con las lamelas secundarias del espiralio. Las anteriores características podrían justificar la pertenencia del género a una nueva subfamilia, incluso a una nueva familia, pero en ausencia de otras formas que, con seguridad, pudieran agruparse en los nuevos taxones (aunque véase luego, en la discusión genérica, Plancella Amsden, 1985), preferimos mantenerlo en la Familia Meristellidae, Subfamilia Meristellinae.

\section{Género Bimeristina n. g.}

Especie-tipo: Bimeristina binnekampi n. sp. (Devónico Inferior, Praguiense).

\section{Diagnosis}

Meristeloideos con yugo provisto de doble conexión del arco yugal con las lamelas primarias y secundarias de los conos espirales. Tallo yugal largo, proyectado posteroventralmente, bifurcado en sendas ramas accesorias que se vuelven sobre sí mismas hasta unirse de nuevo con el tallo formando sendas volutas entre las lamelas primarias y secundarias de los conos, a la manera de Meristella. Ramas laterales secundarias extendiéndose por delante de las ramas laterales primarias hasta conectar el arco yugal con las lamelas secundarias del espiralio.

Meristelloids with the jugal arch connected both with the primary and secondary lamellae of the spiral cones. Long, posteroventrally projected jugal stem, that bifurcates and recurves dorsally, then anteriorly, to reunite with the stem near the start of the lateral jugal branches as in Meristella. Secondary lateral branches starting in front of the primary ones, projecting laterally to join the jugal arch with the secondary lamellae of the spiral cones.

\section{Discusión}

Binnekamp (1965) describió como Whitfieldella spec. una especie abundante en la parte alta de la Formación Lebanza (miembro E), en Palencia. Dicha forma no pertenece al género referido debido al gran desarrollo del septo medio dorsal, que alcanza más de la mitad de la 
a

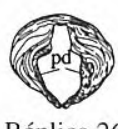

Réplica 263 $(3,8 \mathrm{~mm})$

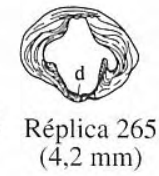

Réplica 254

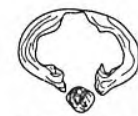

Réplica 269

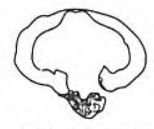

Réplica 272 $(5 \mathrm{~mm})$
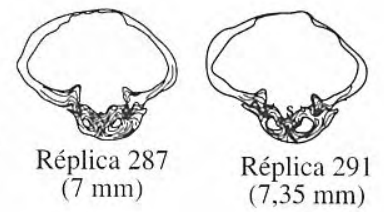
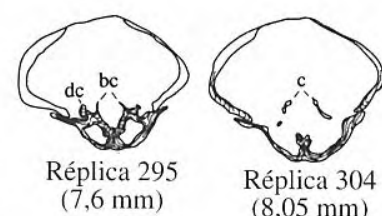

$(8,05 \mathrm{~mm})$
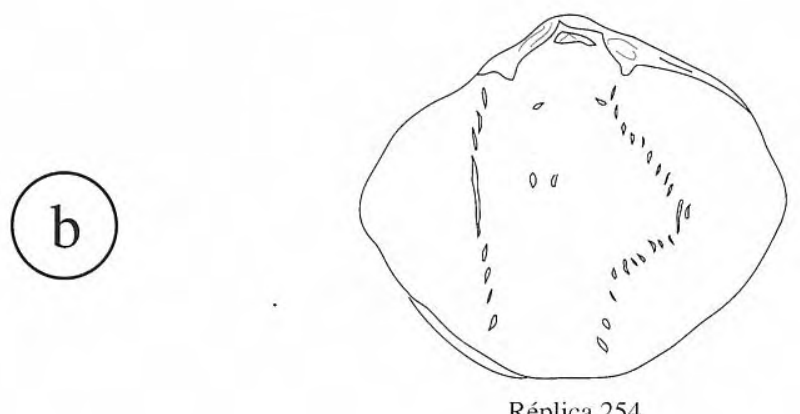

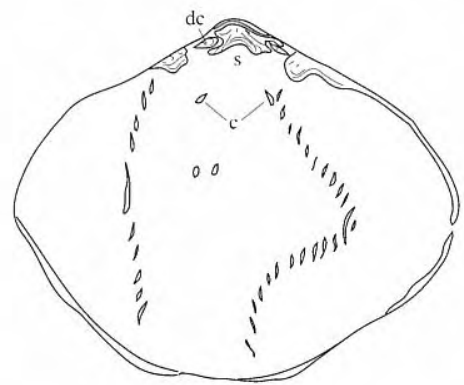

Réplica 259

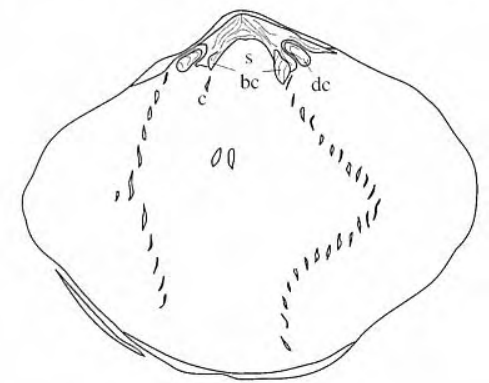

Réplica 262

Figura 4. "Whitfieldella" spec. (colección original de Binnekamp, 1965). Dibujos con cámara clara (aprox. x2). a: Ejemplar RGM 360074 ( $\mathrm{L}=25 \mathrm{~mm}$ ), de Lebanza, parte superior de la Fm. Lebanza. Secciones paralelas, normales a los planos de comisura y de simetría. Distancias en mm al ápice de la valva ventral. b: Ejemplar RGM 360090, de la misma procedencia anterior (no constan dimensiones del ejemplar, ni distancias entre secciones). Serie muy incompleta de secciones paralelas al plano de comisura en sentido ventrodorsal de un ejemplar con aparato yugal en apariencia casi completamente destruído. Abreviaturas igual que en Fig. 5.

"Whitfieldella" spec. (Binnekamp's, 1965, original collection, housed in the Rijksmuseum of Leiden). Camera lucida drawings (ca. x2). a: Specimen RGM 360074 ( $L=25 \mathrm{~mm}$ ), village of Lebanza (Palencia), upper part of the Lebanza Fm. Serial sections normal to commisure and symmetry planes. Distances in mm measured to the ventral valve apex. b: Specimen RGM 360090, of the same procedence as the above specimen (dimensions and distance among sections unknown). Very incomplet series of serial sections ventrodorsally executed parallel to the commisure plane of a specimen with the jugal apparatus in appearance almost completely destroyed. Abbreviations as in Fig. 5 .

longitud de la valva dorsal y tiende a intruir el septalio, la ausencia de placas cardinales cubriendo el septalio y la presencia de tallo yugal bifurcante. El estudio de las secciones seriadas realizadas por Binnekamp y de material nuevo bien conservado de las mismas localidades, recogido por el autor, muestran ciertas características internas próximas a Meristina, como la presencia de placas dentales cortas pero bien desarrolladas y campo muscular ventral poco marcado, pero difieren en la presencia de un aparato braquial bastante más complicado que el descrito por Binnekamp, próximo al de Meristella, cuya originalidad justifica la creación del nuevo género Bimeristina, cuya especie-tipo,
B. binnekampi n. sp. es la antigua Whitfieldella spec. de Binnekamp (1965). Por desgracia, la mayor parte de los numerosos ejemplares seccionados tienen muy dañado el braquidio, de forma que no ha podido comprobarse la expresión del complejo aparato yugal en secciones perpendiculares a los planos de comisura y de simetría.

Los géneros de Triathyrididae, Triathyris Boucot, Johnson y Staton, 1965 y Septathyris Boucot, Johnson y Staton, 1964, del Emsiense, con septalio provisto también de una cresta vertical en su fondo, difieren de Bimeristina, entre otros aspectos, en la presencia de microornamentación externa costulada o de finas líneas plegadas en ángulos agudos dirigidos en dirección

Figura 5. Bimeristina binnekampi n. g., n. sp. Devónico Inferior (Praguiense). a: Dibujos con cámara clara (aprox. x1) del ejemplar DPO 38572 (seccionado; ver Fig. b), procedente de los alrededores de Lebanza (Palencia), yacimiento $\alpha$ 176, de la parte alta de la Fm. Lebanza (miembro E); en ellos se aprecia el desarrollo del septo medio dorsal (Dimensiones: $\mathrm{L}=17 \mathrm{~mm}, \mathrm{a}=16,6 \mathrm{~mm}, \mathrm{~g}=9,5 \mathrm{~mm}$ ). b: Secciones paralelas (todas $\mathrm{x} 2$ ) al plano de la comisura en sentido ventrodorsal del ejemplar anterior. Distancias en milímetros al plano tangente a la valva ventral donde se iniciaron las secciones seriadas. c: Reconstrucción idealizada en vista ventral (aprox. x3) del cardinalio y braquidio de B. binnekampi n. g., n. sp. a partir de las secciones anteriores. Para simplificar sólo se representa parte de la valva y de las espiras del braquidio. d: Secciones transversales a la comisura del ejemplar DPO 38578 procedente de la Cordillera Ibérica Oriental, corte Escalambreras, Fm. Nogueras, yac. A-133 (transición d2c $\alpha$-d2c $\beta$ ) (Dimensiones: $\mathrm{L}=18,35 \mathrm{~mm} ; \mathrm{a}=18,95 \mathrm{~mm} ; \mathrm{g}=12,75 \mathrm{~mm}$ ). Distancias en milímetros al ápice de la valva ventral.

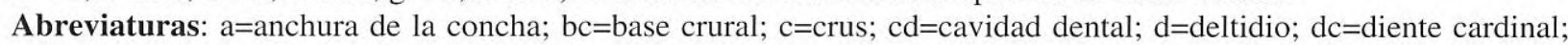
$\mathrm{g}=$ grosor de la concha; $\mathrm{L}=$ longitud de la concha; $\mathrm{l}=$ =lamelas primarias del braquidio; $1 \mathrm{~s}=1$ amelas secundarias del braquidio; $\mathrm{pd}=$ placa dental; $\mathrm{rty}=$ ramas del tallo yugal; $\mathrm{s}=\mathrm{septalio}$; $\mathrm{sm}=$ septo medio; $\mathrm{t}=$ =tallo yugal; $\mathrm{vd}=\mathrm{valva}$ dorsal; via=vista anterior; vid=vista dorsal; vil=vista lateral; vv=valva ventral; $\mathrm{y}=\mathrm{yugo}$. 
posterior y anterior, y en la ausencia de enlace entre el yugo y las lamelas secundarias del espiralio (Álvarez et al., 1998).

El género Plancella Amsden, 1985 tiene estructuras septaliales similares a las de Bimeristina y campo muscular débilmente impreso, pero no se conoce la disposición del braquidio y aparato yugal.

\section{Especies asignadas y a ser investigadas}

Además de la especie-tipo, podría pertenecer al nuevo género la especie Meristella gallei Havlícek (in Havlíček y Vanẻk, 1998), del Praguiense de la República Checa, de la que, por el momento, se desconoce la estructura del aparato yugal.

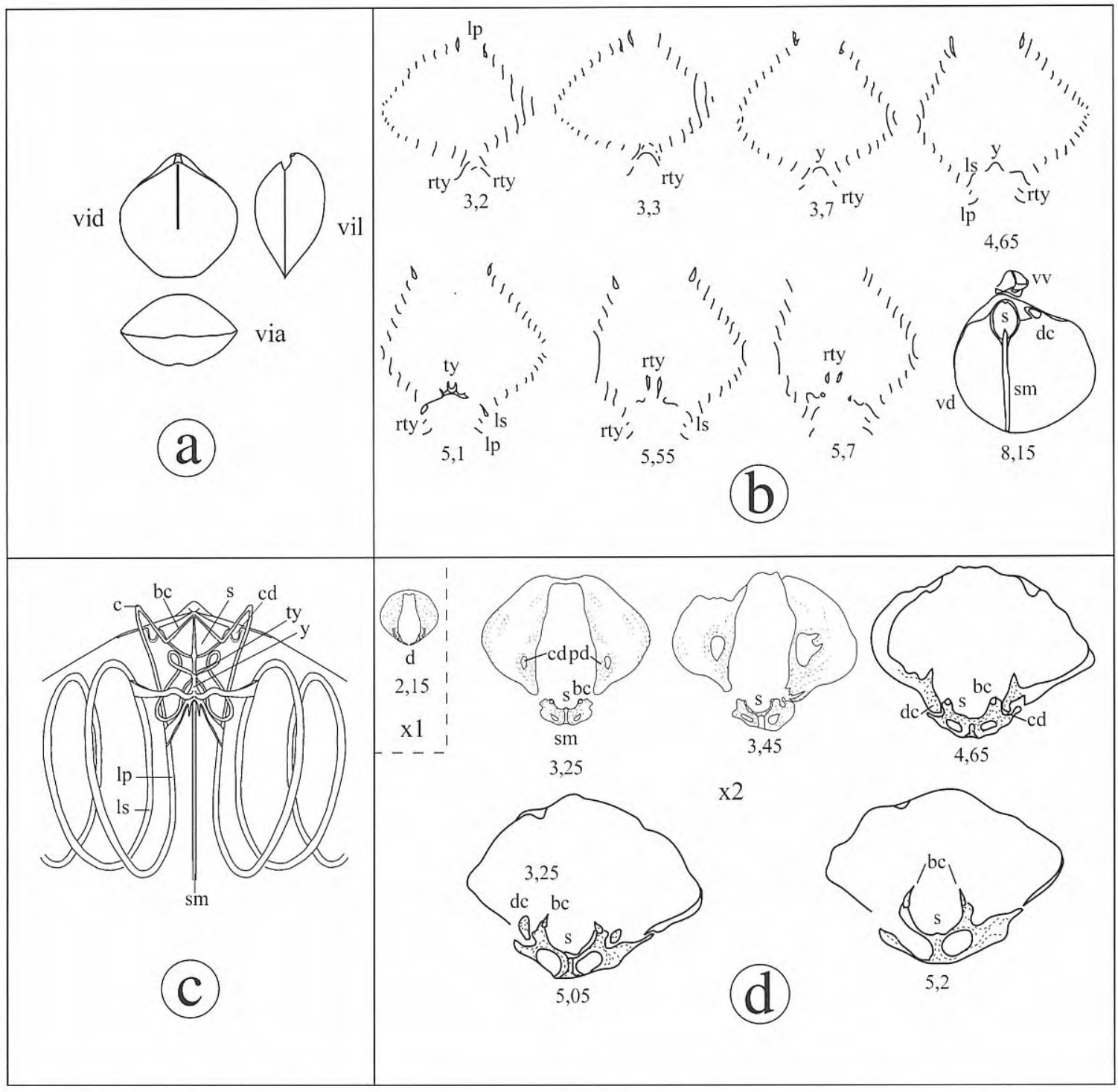

a: Camera lucida drawings (ca. x1) of the specimen DPO 38572 (sectioned; see Fig. b), coming from near Lebanza (Palencia), locality $\alpha-176$ of the upper part of the Lebanza Fm. (E member) showing the long dorsal median septum (Dimensions: $L=17 \mathrm{~mm}, a=16.6 \mathrm{~mm}, \mathrm{~g}=9.5 \mathrm{~mm}$ ). $\boldsymbol{b}$ : Camera lucida drawings $(x 2)$ of serial sections parallel to the commisural plane ventrodorsally executed of the specimen DPO 38572 (see Fig. a). Distances in mm to the ventral valve tangential plane parallel to the commisure. $c$ : Idealized reconstruction in ventral view (ca. $x 3$, based in serial sections of Fig. b) of the B. binnekampi $n$. g., n. sp. cardinalium and brachidium. For sake of simplification only a part of the valve and of the brachial spiral cones have been represented. $d$ : Camera lucida drawings of transversal serial sections of specimen DPO 38578, of the Eastern Iberian Cordillera, Escalambreras section, Nogueras Fm, locality A-133 (d2c $\alpha-d 2 c \beta$ transition) (Dimensions: $L=18.35 \mathrm{~mm} ; a=18.95 \mathrm{~mm} ; \mathrm{g}=12.75 \mathrm{~mm}$ ). Distances in mm to the ventral valve apex.

Abbreviations: $a=$ shell width; $b c=$ crural base; $c=$ crus; $c d=$ dental socket $d=$ deltidium; $d c=$ cardinal tooth; $g=$ shell thickness; L=shell length; lp=brachial primary lamellae; ls=brachial secondary lamellae; pd=dental plate; rty=jugal stem branchs; s=septalium; sm=median septum; ty=jugal stem; $v d=d o r s a l$ valve; via=anterior view; vid=dorsal view; vil=lateral view; vv=ventral valve; $y=j u g u m$. 


\section{Distribución geográfica y estratigráfica}

Cordillera Cantábrica, Dominio Palentino (Fm. Lebanza, miembro E, Praguiense); Cordillera Ibérica Oriental, Aragón (Fm. Nogueras, Praguiense); Guadarrama oriental, Guadalajara (Fm. Cercadillo, Praguiense).

\section{Bimeristina binnekampi n. sp. Figs.1-6, Tab. 1}

v. 1965 Whitfieldella spec.; Binnekamp, 39, lám. 9, figs. 14a, fig.text. 23 .

Derivatio nominis: Especie dedicada al Dr. J.G. Binnekamp, antiguo investigador del Rijksmuseum van Geologie en Mineralogie de Leiden (Holanda), en homenaje a su excelente trabajo pionero sobre la fauna de braquiópodos devónicos de Palencia.

\section{Material}

Holotipo DPO 30895 (Fig. 2a-d) y 33 Paratipos, DPO 30980 (Fig. 2e-h), 30863 (Fig. 2i-1), 30865-30894 y 31317 (seccionado, pero no representado aquí), procedentes del estrato y localidad típicos. Otros 3 ejemplares, procedentes de diferentes niveles de la localidad-tipo, DPO 30862 de $\alpha$ 247 (antiguo M-LEB-I-47) y DPO 31347-31348, de $\alpha-255$ (antiguo M-LEB-I-55). Un ejemplar, DPO 38569, de la ladera septentrional del pico Cuchillón, al E de la abadía de Lebanza, yac. 1-78 (1988). 12 ejemplares, DPO 30853 30861, 38570 (seccionado, Fig. 3a), 38571 y 38572 (seccionado, Fig. 5a-c), de la parte alta de la Fm. Lebanza, miembro E, yac. $\alpha-176$, del camino de carros que sale de la carretera de Lebanza a la abadía de Lebanza, al OSO de aquel pueblo.

Otros 5 ejemplares, DPO 38573-38576 y 38577 (Fig. $2 \mathrm{~m}-\mathrm{o}$ ), procedentes del Guadarrama oriental, niveles C3 a C4 (=MS 11/12=A-149=A-201) de la Fm. Cercadillo, y 10 ejemplares, DPO 33969 (Fig 2p-s)-33971, 34132-34136, 36050 y 38578 (seccionado, Fig. 5d) de la Cordillera Ibérica Oriental, de la Fm. Nogueras, transición entre los niveles $\mathrm{d} 2 \mathrm{c} \alpha$ y d2c $\beta$ (ver versión actualizada de la estratigrafía del Guadarrama oriental y Cordillera Ibérica oriental en GarcíaAlcalde et al., 2002).

Para el presente estudio, se analizaron también y representaron secciones seriadas originales de Binnekamp (1965) de los ejemplares depositados en el Rijksmuseum van Geologie en Mineralogie de Leiden (Holanda), con los números RGM 360055 (reproducidas, en parte, en Fig. 3b, tres de las cuales, 248,250 y 251 están figuradas incompletas, en Binnekamp, 1965, Fig. 23), RGM 360074 (reproducidas, en parte, en Fig. $4 \mathrm{a}$, una de las cuales, la $\mathrm{n}^{\circ}$ 287 fue publicada por Binnekamp, 1965, pl. 9, fig. 4a), RGM 360090 (Fig. 4b) y RGM 360091-360093.

\section{Estrato y localidad típicos}

Calizas packstone y wackestone con finos lechos de lutitas carbonatadas oscuras intercaladas, de la parte alta de la Formación Lebanza (miembro E), perteneciente al intervalo faunístico 6 de García-Alcalde (1996), del Praguiense. Nivel $\alpha-252 / 253$ (antiguo M-LEB-I-52/53B).
Corte estratotípico de la Fm. Lebanza, llamado de "Las Cabañinas", unos $900 \mathrm{~m}$ al OSO del pueblo de Lebanza (Palencia) (Fig. 1).

\section{Diagnosis}

Concha de tamaño pequeño a medio, de contorno pentagonal redondeado, equidimensional, débilmente ventribiconvexa. Ambas valvas provistas de una débil depresión longitudinal media; comisura frontal rectimarginada o casi. Septo medio dorsal muy largo, extendiéndose más allá de la mitad de la longitud.

Rounded pentagonal, faintly biconvex, small- to median-sized shell, as long as wide. Shallow and narrow longitudinal median sinuses developed on both valves; frontal commisure rectimarginate or nearly so. Dorsal median septum very long reaching further than the halflength.

\section{Descripción}

Concha lisa, distrófica, de tamaño pequeño a medio (L comprendido entre 11 y $23,4 \mathrm{~mm}$ en el material mejor conservado; Tab. 1), contorno pentagonal redondeado, equidimensional o casi (media de $\mathrm{a} / \mathrm{L}$ en el material mejor conservado=0,96; Tab. 1 y Fig. 6), débilmente ventribiconvexa, casi dos veces más larga que gruesa (media de $\mathrm{g} / \mathrm{L}$ en el material mejor conservado=0,58; Tab. 1 y Fig. 6). La máxima anchura de la concha se sitúa por delante de la charnela, en la mitad anterior de la longitud (por término medio hacia el 55\% de L; Tab. 1). Curvatura de ambas valvas, en vistas lateral y anterior, regular, encontrándose los bordes de ambas valvas en las comisuras según ángulos agudos, salvo en los ejemplares gerónticos donde forman ángulos obtusos no muy alejados del recto (Fig. 2s). Máximo grosor de la valva ventral en la región umbonal; máximo grosor de la dorsal hacia la mitad de la longitud. Superficie de ambas valvas recorrida por una débil depresión media, algo más marcada en la ventral, de manera que la comisura frontal es rectimarginada o débilmente desviada en dirección dorsal.

Gancho ventral suberecto a recurvado en los ejemplares de mayor edad. Foramen peduncular grande, circular, mesotírido. Deltirio parcialmente cubierto por un deltidio grueso y arqueado, situado bajo el gancho (Fig. 4a). Ángulo apical superior a un recto $\left(95^{\circ}\right.$ de media en los ejemplares mejor conservados; Tab. 1). Aristas umbonales rectas, más o menos acusadas, extendiéndose desde el gancho hasta los extremos de la charnela.

Superficie de la concha recorrida por líneas concéntricas de crecimiento finas, carentes de expansiones lamelares.

Interior de la valva ventral con placas dentales cortas, fuertes, con caras internas cóncavas; cavidades laterales ocupadas en parte por macizos callotestarios, sobre todo en los ejemplares maduros (Fig. 4a, 5d). Dientes cardinales cortos y macizos, de bases débilmente excavadas para acomodar los extremos superiores de los rebordes internos de las cavidades dentales, formando un mecanismo articulatorio complementario (Figs. 3, 4). Campo muscular triangular y alargado, débilmente impreso, recorrido, igual que el resto de la concha, por finas elevaciones radiales (Fig. 2h). El campo muscular se extiende por delante de un 

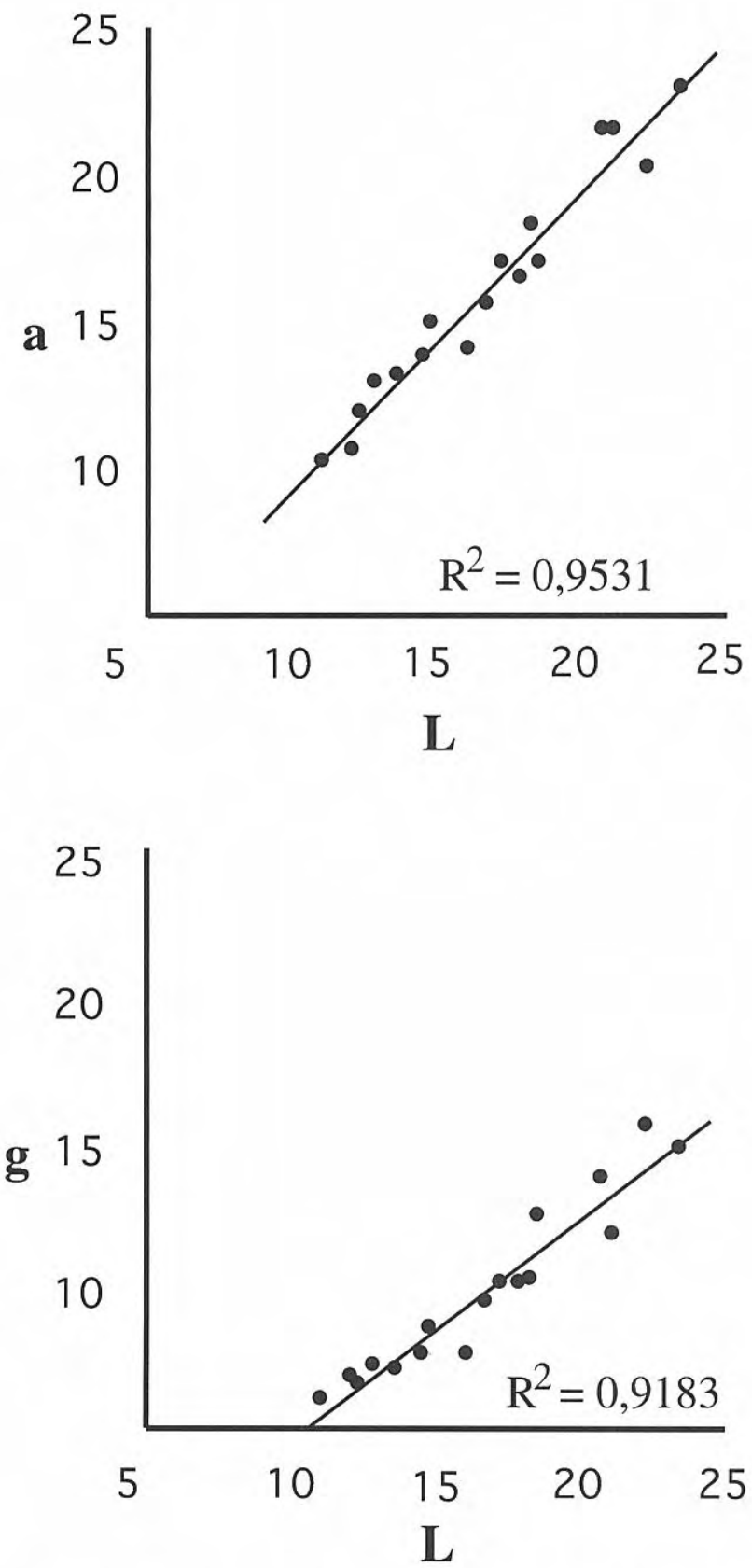

Figura 6. Diagrama de dispersión longitud/anchura (L/a) y longitud/grosor $(\mathrm{L} / \mathrm{g})$ de Bimeristina binnekampi n. g., n. sp.

Length/Width (L/a) and Length/Thickness $(\mathrm{L} / \mathrm{g})$ dispersion diagrams of Bimeristina binnekampi $n$. g., n. sp.

pequeño macizo callotestario desarrollado en el ápice de la valva.

Interior de la valva dorsal con septalio abierto en forma de $U$ y fondo parcialmente ocupado por macizos callotestarios en los ejemplares adultos (Fig. 4a), soportado por un septo medio, bajo y muy largo (más del $50 \%$ de la longitud de la valva dorsal; Tab. 1 y Figs. 2-5), que parece prolongarse en el fondo del septalio en un pequeño resalte vertical, más o menos desarrollado (Figs. 3-5). Cavidades dentales estrechas; rebordes internos de las cavidades, robustos, con extremos superiores encajados en las depresiones de las bases de los dientes cardinales (Fig. 3-5). Bases crurales proyectándose casi verticalmente, formando extensiones agudas cerca del extremo anterior del septalio (Fig. 3-5) y prolongándose luego en cruras finos que se dirigen ventralmente hasta unirse con las lamelas primarias del espiralio. Conos espirales constituidos por 12 a 13 circunvoluciones dirigidas lateralmente (Figs. 4b, 5b). Yugo constituido por un arco yugal dirigido posteroventralmente, bifurcado en sendas ramas yugales accesorias que se prolongan hacia atrás, recurvándose luego sobre sí mismas para formar volutas cuyos extremos se unen de nuevo al arco yugal, cerca del arranque de las ramas laterales; el arco yugal se alarga anteriormente en pequeñas aletas simulando una silla yugal incipiente. Las ramas laterales se extienden anterodorsalmente, conectando el yugo con las lamelas primarias, pero existen también otras ramas suplementarias, inmediatamente delante de las laterales, que se proyectan hacia los lados, conectando el yugo con las lamelas secundarias del espiralio (Fig. 5b-c). Campo muscular dorsal débilmente impreso, alargado y lanceolado, bisectado por el septo medio (Fig. 2k).

El cierre de la concha, por delante de la región cardinal, se efectúa mediante el solapamiento de extensiones de la valva ventral por otras de la dorsal (Figs. 3-4), similares a las glottae y squamae, frecuentes en muchos rinconélidos (Westbroek, 1967).

\section{Discusión}

La apreciación de un yugo en forma de Y (tallo yugal proyectado posteroventralmente y bifurcado en sendas ramas accesorias), en ejemplares probablemente dañados e incompletos (lo que es habitual en el material original de la especie), hizo que Binnekamp (1965), aproximase la forma descrita al género Whitfieldella, muy distinto en su morfología interna a Bimeristina, como ya se indicó en la discusión genérica.

Meristella gallei Havlícek (in Havlícek y Vanèk, 1998), difiere en el tamaño mayor, carácter alargado de la concha, mayor grosor, menor ángulo apical, comisura frontal netamente uniplegada, campo muscular ventral claramente impreso y septo medio dorsal mucho más grueso.

Plancella turkiensis Amsden, 1985, es más pequeña, alargada y gruesa, de contorno triangular, menor ángulo apical, con plegamiento alternante caracterizado por la presencia de un débil pliegue medio en la valva dorsal y septalio mucho más somero.

\section{Distribución geográfica y estratigráfica \\ La misma que para el género.}

\section{AGRADECIMIENTOS}

Mi más profundo reconocimiento al Dr. C.F. WinklerPrins, por el préstamo de las preparaciones de las secciones seriadas originalmente realizadas sobre Whitfieldella spec. por Binnekamp (1965), en parte redibujadas para ilustrar el presente trabajo. 
Agradezco a los Drs. Fernando Álvarez, de la Universidad de Oviedo y Miguel Pardo y Rodolfo Gozalo, de la Universidad de Valencia, la minuciosa revisión del manuscrito, que ha mejorado mucho gracias a sus correcciones y recomendaciones.

Esta publicación se desarrolló en el contexto del Proyecto Básico de Investigación de la Dirección General de Enseñanza Superior e Investigación Científica PB 98/1542 "Geoquímica y Geofísica aplicadas a la investigación paleogeográfica, cronoestratigráfica y de bioeventos del Devónico del Macizo Ibérica (España)".

\section{BIBLIOGRAFÍA}

Álvarez, F. 1990. Devonian Athyrid brachiopods from the Cantabrian Zone (NW Spain). Biostratigraphie $d u$ Paléozö̈que, 11, 1-311.

Álvarez, F., Rong, J.-Y. and Boucot, A.J. 1998. The classification of athyridid brachiopods. Journal of Paleontology, 72, 827-855.

Amsden, T. W. 1985. Brachiopods from the Turkey Creek Limestone (Early Devonian) Marshall County, Southern Oklahoma. Oklahoma Geological Survey, Bulletin, 138, $1-20$.

Binnekamp, J. 1965. Lower Devonian brachiopods and stratigraphy of North Palencia (Cantabrian Mountains, Spain). Leidse Geologische Mededelingen, 33, 1-62.

Boucot, A.J., Johnson, J.G. and Staton, R.D. 1964. On some atrypoid, retzioid, and athyridoid Brachiopoda. Journal of Paleontology, 38, 805-822.

Boucot, A.J., Johnson, J.G. and Staton, R.D. 1965 Suborders Retziidina and Athyrididina. In: Treatise on Invertebrate Paleontology, part $\mathrm{H}$, Brachiopoda. Geological Society of America and University of Kansas Press, New York \& Lawrence, 2, H649-H667.

García-Alcalde, J.L. 1996. El Devónico del Dominio AsturLeonés en la Zona Cantábrica (N de España). Revista Española de Paleontología, $\mathbf{n}^{\mathbf{0}}$ extraordinario, 58-71.
García-Alcalde, J.L. 1998. Braquiópodos rinconélidos del Lochkoviense (Devónico Inferior) de la Cordillera Cantábrica (Norte de España). Geobios, 31, 767-789.

García-Alcalde, J.L. 1999. Nuevo género de braquiópodos rinconélidos del Praguiense (Devónico Inferior) de la región Cántabro-Celtibérica (España). Revista Española de Paleontología, 14, 83-91.

García-Alcalde, J.L., Montesinos, J.R., Truyols-Massoni, M., García-López, S., Arbizu, M.A. and Soto, F. 1990. The Palentine Domain (Palentian Zone). In: PreMesozoic Geology of Iberia (Eds. R.D. Dallmeyer and E. Martínez-García), Springer Verlag, 2 (2) Cantabrian and Palentine Zones: Stratigraphy, 20-23.

García-Alcalde, J.L., Carls, P., Pardo Alonso, M.V., Sanz López, J., Soto, F., Truyols-Massoni, M. and Valenzuela-Ríos, J.I. 2002. Chapter 6: Devonian. In: The Geology of Spain (Eds. W. Gibbons and T. Moreno). Geological Society, London, 67-91.

García-López, S., Jahnke, H. and Sanz-López, J. 2002. Uppermost Prídolí to Upper Emsian stratigraphy of the Alto Carrión Unit, Palentine Domain (Northwest Spain). In: Palaeozoic Conodonts from northern Spain (Eds. S. García-López and F. Bastida). Cuadernos del Museo Geominero, 1, 229-257.

Hall, J. 1859. Observations on genera of Brachiopoda. 12th Annual Report New York State Cabinet, 8-110.

Havlícek, V. and Vanèk, J. 1998. Pragian brachiopods, trilobites, and principal biofacies in the Prague Basin (Lower Devonian, Bohemia). Sborník geologickyck ved, Palaeontologie, 34, 27-109.

Krans, Th.F., Guit, F.A. and Ofwegen, L.P. van. 1982. Facies-patterns in the Lower Devonian carbonates of the Lebanza Formation (Cantabrian Mountains, Province of Palencia, NW Spain). Neues Jahrbuch Geologie und Paläontologie Abhandlungen, 163, 192-230.

Westbroek, P. 1967. Morphological observations with systematic implications on some Palaeozoic Rhynchonellida from Europe with special emphasis on the Uncinulidae. Leidse Geologische Mededelingen, 41, $1-82$. 